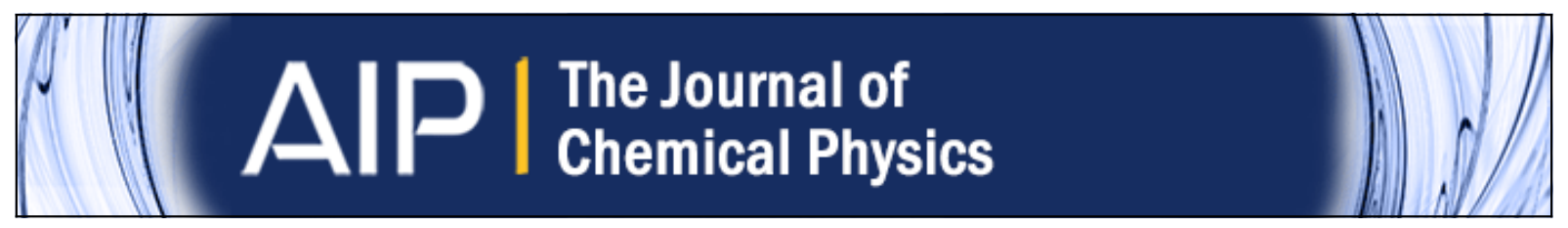

Quantum effects in the capture of charged particles by dipolar polarizable symmetric top molecules. II. Interplay between electrostatic and gyroscopic interactions

M. Auzinsh, E. I. Dashevskaya, I. Litvin, E. E. Nikitin, and J. Troe

Citation: The Journal of Chemical Physics 139, 144315 (2013); doi: 10.1063/1.4821589

View online: http://dx.doi.org/10.1063/1.4821589

View Table of Contents: http://scitation.aip.org/content/aip/journal/jcp/139/14?ver=pdfcov

Published by the AIP Publishing

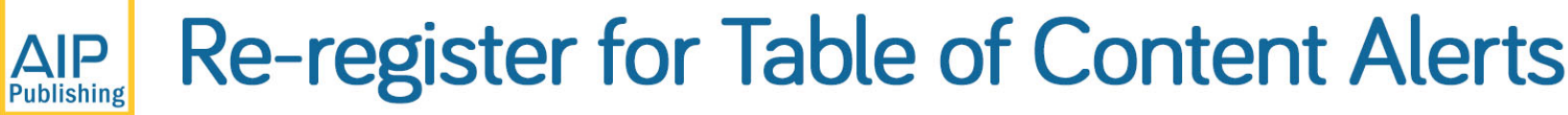

\section{Create a profile.}




\title{
Quantum effects in the capture of charged particles by dipolar polarizable symmetric top molecules. II. Interplay between electrostatic and gyroscopic interactions
}

\author{
M. Auzinsh, ${ }^{1}$ E. I. Dashevskaya, ${ }^{2,3}$ I. Litvin, ${ }^{3}$ E. E. Nikitin, ${ }^{2,3}$ and J. Troe ${ }^{3,4, a)}$ \\ ${ }^{1}$ Department of Physics, University of Latvia, Riga LV-1586, Latvia \\ ${ }^{2}$ Schulich Faculty of Chemistry, Technion - Israel Institute of Technology, Haifa, 32000, Israel \\ ${ }^{3}$ Max-Planck-Institut für Biophysikalische Chemie, Am Fassberg 11, Göttingen D-37077, Germany \\ ${ }^{4}$ Institut für Physikalische Chemie, Universität Göttingen, Tammannstrasse 6, Göttingen D-37077, Germany
}

(Received 7 August 2013; accepted 4 September 2013; published online 10 October 2013)

\begin{abstract}
Within the general axially nonadiabatic channel approach described in Paper I of this series [M. Auzinsh, E. I. Dashevskaya, I. Litvin, E. E. Nikitin, and J. Troe, J. Chem. Phys. 139, 084311 (2013)], the present article analyzes the simultaneous manifestation of electrostatic and gyroscopic interactions in the quantum capture of dipolar polarizable symmetric top molecules by ions. As a demonstration, the rate coefficients for capture of $\mathrm{CH}_{3} \mathrm{D}$ and $\mathrm{CD}_{3} \mathrm{H}$ by $\mathrm{H}^{+}, \mathrm{D}^{+}$, and $\mathrm{H}_{3}{ }^{+}$are calculated. (C) 2013 AIP Publishing LLC. [http://dx.doi.org/10.1063/1.4821589]
\end{abstract}

\section{INTRODUCTION}

In Paper $\mathrm{I}^{1}$ of this series we have presented a general axially nonadiabatic channel treatment of the capture of charged particles by dipolar polarizable symmetric top molecules with the aim to reveal quantum effects in the collision dynamics. In general, these effects are related to the discrete nature of the intrinsic, orbital, and total angular momenta, to the quantum character of passage of collision partners across effective potential barriers and drops, and to the interplay of two types of anisotropic interactions, the gyroscopic (Coriolis) and the electrostatic ones. The latter feature, in principle, leads to a coupling of capture channels. In the calculation of capture cross sections or rate coefficients, however, this coupling can be ignored provided that the reorientation of the intrinsic angular momenta from the spacefixed axis onto the body-fixed axis occurs at larger interfragment distances than those essential for capture. This is the case when the interaction with increasing intermolecular distance falls off faster than the centrifugal potential (and the Coriolis interaction), thus permitting the use of the standard adiabatic channel treatment of Refs. 2-4. An extension of this treatment to very low collision energies, where a small number of partial waves contribute to the capture, was done for anisotropic charge-induced dipole interaction, ${ }^{5}$ resonance dipole-dipole interaction, ${ }^{6,7}$ and charge-quadrupole interaction. ${ }^{8}$ In all these cases, the energy-dependent capture rate coefficient approaches a constant value at zero-energy, i.e., it conforms with the Bethe law. ${ }^{9}$

For an ion and a dipolar molecule possessing a nonzero average dipole moment in a given rotational state, the first-order charge-dipole interaction falls off as the centrifugal interaction. This results in a divergence of the capture rate coefficient in the zero-energy limit, i.e., in a violation of the Bethe law. Here, the quantum effects mentioned above are supplemented by the partial locking of the intrinsic an-

\footnotetext{
a)E-mail: shoff@gwdg.de
}

gular momentum $\mathbf{j}$ to the collision axis and the interplay of charge-dipole (electrostatic) and Coriolis (gyroscopic) interactions. It was suggested in Ref. 10 that a physical system, for which all quantum effects show up, is given by the capture of isotopically substituted methane molecules by ions. This work further pursues this point on the basis of the general properties of axially nonadiabatic (ANC) capture rate coefficients and it presents calculations of energy- and temperaturedependent rate coefficients. We concentrate here on the low energy/temperature collision regime, when only few partial waves contribute to the capture such that our results bridge the gap between the fly-wheel limit (single-wave capture and very small dipole moments) and the classical adiabatic channel limit (many-wave capture and large dipole moments).

The plan of the paper is as follows. In Sec. II we define dimensionless scaled parameters that control the capture dynamics. Section III provides a general discussion of energy- and temperature-dependent capture rate coefficients for symmetric tops with small dipole moments. Section IV contains a qualitative discussion of $K$-doubling effects in the capture. Section V presents calculations for capture of $\mathrm{CH}_{3} \mathrm{D}$ and $\mathrm{CD}_{3} \mathrm{H}$ molecules by a series of ions. Section VI concludes the paper.

\section{KEY PARAMETERS RELEVANT FOR CAPTURE}

Molecular parameters that govern the capture dynamics of polarizable dipolar symmetric top rotors by ions in the adiabatic channel weak-field approximation are the relative collision energy $E$ (or the translational temperature $T$ ), the rotational state $j, k$ of the top, the reduced mass of the colliding partners $\mu$, the charge of the ion $q$, the polarizability $\alpha$, and the dipole moment $\mu_{D}$ of the neutral top. Combination of these parameters into two dimensionless quantities defines a reduced collision energy $\varepsilon$ (or reduced temperature $\theta$ ) and a reduced dipole moment $\delta$ :

$$
\varepsilon=E / E_{L} \text { and } \theta=k_{\mathrm{B}} T / E_{L} \quad \text { with } E_{L}=\hbar^{4} / \mu^{2} q^{2} \alpha
$$


and

$$
\delta=q \mu \bar{\mu}_{D} / \hbar^{2} \quad \text { with } \bar{\mu}_{D}=\mu_{D} k / \sqrt{j(j+1)} .
$$

ANC capture rate coefficients are scaled with respect to the Langevin rate coefficient $k_{\text {Lang }}=2 \pi q \sqrt{\alpha / \mu}$. For a top with the rotational quantum number $j$, then they are expressed either through $\varepsilon$ and $\delta$ (denoted by ${ }^{\mathrm{NNC}} \chi^{(j)}(\varepsilon, \delta)$ ) or through $\theta$ and $\delta$ (denoted by ${ }^{\mathrm{ANC}} \bar{\chi}^{(j)}(\theta, \delta)$ ).

In what follows we discuss the capture of a symmetric top of structure $\mathrm{XY}_{3} \mathrm{Y}^{\prime}$ that originates from a spherical top $\mathrm{XY}_{4}$ by substitution of the atom $\mathrm{Y}$ by an isotope $\mathrm{Y}^{\prime}$. As discussed earlier ${ }^{10}$ the nonzero dipole moment of $\mathrm{XY}_{3} \mathrm{Y}^{\prime}$ in its ground vibrational state is due to the difference in the mean dipole moments of the $\mathrm{XY}$ and $\mathrm{XY}^{\prime}$ bonds. Qualitatively, this difference arises from different mean lengths of the bonds through anharmonicity of the vibrational motion in the ground vibrational state. As outlined in Ref. 10 for a Morse model of the isolated XY bond of length $r$ (with conventional Morse parameters $r_{e}, \beta, D$ ) and a small-amplitude frequency $\omega_{e}$, the dipole moment $\mu_{D}$ of the $\mathrm{XY}_{3} \mathrm{Y}^{\prime}$ top in its ground vibrational state is proportional to the difference $\left|\omega_{e}-\omega_{e}^{\prime}\right|$ of the frequencies of the $\mathrm{XY}$ and $\mathrm{XY}^{\prime}$ Morse oscillators. This observation allows one to establish a qualitative dependence of the parameter $\delta$ on the reduced mass $\mu$ of the colliding partners $\mathrm{XY}_{3} \mathrm{Y}^{\prime}$ and $\mathrm{Z}^{+}$and the reduced masses $m$ and $m^{\prime}$ of the $\mathrm{XY}$ and $X \mathrm{Y}^{\prime}$ oscillators:

$$
\delta \propto \mu\left|\frac{1}{\sqrt{m}}-\frac{1}{\sqrt{m^{\prime}}}\right| .
$$

With different $\mathrm{X}, \mathrm{Y}, \mathrm{Y}^{\prime}, \mathrm{Z}^{+}$moieties, the first factor of the r.h.s. of Eq. (2.3) can vary from about unity (when $\mathrm{Z}^{+}=\mathrm{H}^{+}$) to values markedly exceeding unity (when $\mathrm{Z}^{+}$is much heavier than $\mathrm{H}^{+}$), while the second factor may decrease from about unity (when $\mathrm{Y}=\mathrm{H}$ and $\mathrm{Y}^{\prime}=\mathrm{D}$ ) to very small values (when $\mathrm{Y}$ and $\mathrm{Y}^{\prime}$ are much heavier than $\mathrm{H}$ and $\mathrm{D}$ ). The interplay of these two trends results in a wide range of possible values of $\delta$, including those for which interesting quantum effects in the capture are expected. It should be mentioned that the crude Morse model of independent bonds yields the correct order of magnitude of $\mu_{D}$ for $\mathrm{CH}_{3} \mathrm{D},{ }^{10}$ see also Sec. V.

\section{ACCI RATE COEFFICIENTS}

As a reference rate coefficient for discussion of the quantum and gyroscopic effects in the capture of dipolar molecules by ions, we take the $\mathrm{ACCl}$ rate coefficient which provides a good approximation to accurate rate coefficients under the condition $\varepsilon \gg 1$ and $\delta \gg 1$ with the relative accuracy of $1 / J_{c}$, where $J_{c}$ is a characteristic value of the total angular momentum for the capture event. In what follows we use the scaled (to its Langevin counterpart) $\mathrm{ACCl}$ rate coefficient, ${ }^{\mathrm{ACCl}} \chi^{(j)}(\varepsilon, \delta)$, which was used in earlier work on capture in the classical regime. An additional reason for using ${ }^{\mathrm{ACCl}} \chi^{(j)}(\varepsilon, \delta)$ as a reference, beside its property to provide correct asymptotic ANC rate coefficients, ${ }^{\mathrm{ANC}} \chi^{(j)}(\varepsilon, \delta)$, is its feature to coincide with the latter in the limit $\varepsilon \ll 1$ for certain values of $\delta, \delta={ }^{\mathrm{R}} \delta_{n}^{(J, j)}$, which in Paper $\mathrm{I}^{1}$ was called the reference set. In this way, one could expect that the energy-dependent and temperature-dependent ratios of the $\mathrm{ANC} / \mathrm{ACCl}$ rate coeffi- cients would not be too different from unity across a wide range of energy/temperature, from $\varepsilon, \theta \ll 1$ to $\varepsilon, \theta \gg 1$, though in the former case the $\mathrm{ACCl}$ rates have to be extrapolated beyond their formal limits of validity.

The quantities ${ }^{\mathrm{ACCl}} \chi^{(j)}(\kappa, \delta)$ are given by ${ }^{1}$

$$
\begin{aligned}
{ }^{\mathrm{ACCl}} \chi^{(j)}(\kappa, \delta)= & \frac{1}{2 j+1} \sum_{n=-j}^{j}\left(1+\frac{n \delta}{\kappa \sqrt{j(j+1)}}\right) \\
& \times \Theta\left(1+\frac{n \delta}{\kappa \sqrt{j(j+1)}}\right),
\end{aligned}
$$

where $\Theta(x)$ denotes the step function of $x$. The quantities ${ }^{\mathrm{ACCl}} \chi^{(j)}(\kappa, \delta)$ are expressed as a function of the ratio $\delta / \sqrt{2 \varepsilon}$ by

$$
\begin{aligned}
{ }^{\mathrm{ACCl}} \chi^{(j)}(\varepsilon, \delta) \equiv & { }^{\mathrm{ACCl}} \chi^{(j)}(\delta / \sqrt{2 \varepsilon}) \\
= & \frac{1}{2 j+1} \sum_{n=-j}^{j}\left(1+\frac{n \delta}{\sqrt{2 \varepsilon} \sqrt{j(j+1)}}\right) \\
& \times \Theta\left(1+\frac{n \delta}{\sqrt{2 \varepsilon} \sqrt{j(j+1)}}\right) .
\end{aligned}
$$

The averaged scaled capture rate coefficient, ${ }^{\mathrm{ACCl}} \chi^{(j)}(\theta, \delta)$, expressed as a function of the scaled temperature $\theta=k_{B} T / E_{L}$, finally reads

$$
{ }^{\mathrm{ACCl}} \bar{\chi}^{(j)}(\theta, \delta)=\int_{0}^{\infty}{ }^{\mathrm{ACCl}} \chi^{(j)}(\varepsilon, \delta) F(\varepsilon, \theta) d \varepsilon
$$

with the Boltzmann distribution

$$
F(\varepsilon, \theta)=\frac{2}{\theta^{3 / 2} \sqrt{\pi}} \exp (-\varepsilon / \theta) \sqrt{\varepsilon} .
$$

The quantity ${ }^{\mathrm{ACCl}} \bar{\chi}^{(j)}(\theta, \delta)$ is expressed analytically as a function of the single parameter $\delta_{\theta}=\delta / \sqrt{2 \theta}$

$$
\begin{aligned}
{ }^{\mathrm{ACCl}} \bar{\chi}^{(j)}(\theta, \delta) \equiv & { }^{\mathrm{ACCl}} \bar{\chi}^{(j)}\left(\delta_{\theta}\right) \\
= & 1+\frac{\delta_{\theta}}{2 \sqrt{\pi}} \frac{\sqrt{j(j+1)}}{j+1 / 2} \\
& -\frac{1}{2 j+1} \sum_{n=1}^{j} \operatorname{erf}\left(\frac{\delta_{\theta} n}{\sqrt{j(j+1)}}\right),
\end{aligned}
$$

where $\operatorname{erf}(x)$ is the error function. For $j \gg 1$, Eq. (3.3) transforms into

$$
{ }^{\mathrm{ACCl}} \bar{\chi}^{(j \gg 1)}\left(\delta_{\theta}\right)=1+\frac{\delta_{\theta}}{2 \sqrt{\pi}}+\frac{1-\exp \left(-\delta_{\theta}^{2}\right)}{2 \sqrt{\pi} \delta_{\theta}}-\frac{1}{2} \operatorname{Erf}\left(\delta_{\theta}\right) .
$$

Figure 1 shows the plot of ${ }^{\mathrm{ACCl}} \bar{\chi}^{(j \gg 1)}\left(\delta_{\theta}\right)$, plots of two asymptotics (the Langevin and ACClCD capture rate coefficients) (left ordinate axis) and plots of the ratios ${ }^{\mathrm{ACCl}} \bar{\chi}^{(j)}\left(\delta_{\theta}\right){ }^{\mathrm{ACCl}} \bar{\chi}^{(j \gg 1)}\left(\delta_{\theta}\right)$ (right ordinate axis) as a function of the scaled temperature parameter $2 \theta / \delta^{2}=\delta_{\theta}^{-2}$. The latter illustrates the effect of finite values of $j$ on the ACCl rate coefficient. As expected, that effect begins to show up when the charge-dipole interaction noticeably modifies the Langevin capture rate. 


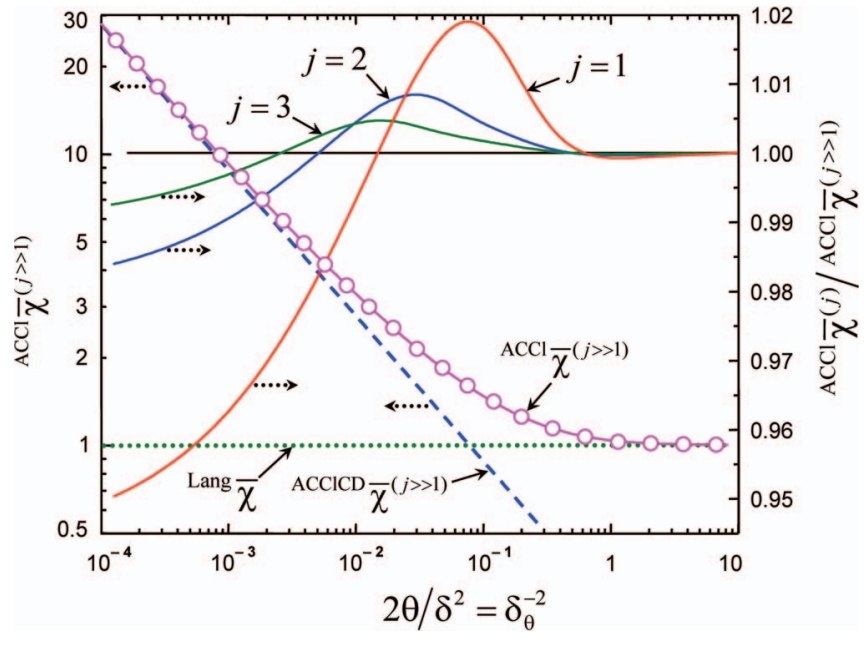

FIG. 1. Plot of ${ }^{\mathrm{ACCl}} \bar{\chi}^{(j \gg 1)}$ (symbols), plots of its two asymptotics (the Langevin, dotted line, and ACClCD, dashed line, capture rate coefficients) (left ordinate axis), and plots of the ratios ${ }^{\mathrm{ACCl}} \bar{\chi}^{(j)} /{ }^{\mathrm{ACCl}} \bar{\chi}^{(j \gg 1)}$ (right ordinate axis) vs. scaled temperature $2 \theta / \delta^{2}=\delta_{\theta}^{-2}$.

\section{ANC RATE COEFFICIENTS}

The ANC capture rate coefficient ${ }^{\mathrm{ANC}} \chi \chi^{(j)}(\varepsilon, \delta)$ is expressed through the capture probability ${ }^{\mathrm{ANC}} P_{n}^{(J, j)}(\varepsilon, \delta)$ as

$$
{ }^{\mathrm{ANC}} \chi^{(j)}(\varepsilon, \delta)=\sum_{J, n} \frac{2 J+1}{2 j+1}{ }^{\mathrm{ANC}} P_{n}^{(J, j)}(\varepsilon, \delta)
$$

where the ${ }^{\mathrm{ANC}} P_{n}^{(J, j)}(\varepsilon, \delta)$ are recovered from the solution of the capture equation that contains the eigenvalues, $c={ }^{\mathrm{ANC}} c_{n}^{(J, j)}(\delta)$, of the ANC interaction matrix, ${ }^{\mathrm{ANC}} \mathbf{C}^{(J, j)}(\delta)$, see Paper I. ${ }^{1}$ The capture channels (each specified by a triad $(J, j, n))$, that at the energy $\varepsilon$ noticeably contribute to the sum in Eq. (3.1), can roughly be identified by the classical condition that their effective potential barriers (if they exist), created by the anisotropic weak-field charge-dipole and gyroscopic interactions on the background of the isotropic chargeinduced dipole interaction, are below $\varepsilon$. This condition reads ${ }^{\mathrm{ANC}} c_{n}^{(J, j)}(\delta) \leq \sqrt{8 \varepsilon}$; for a given $\varepsilon$, it provides an upper limit to positive values of ${ }^{\mathrm{ANC} C} c_{n}^{(J, j)}(\delta)$ and has no effect on negative values of ${ }^{\mathrm{ANC}} c_{n}^{(J, j)}(\delta)$. As an example, Fig. 2 shows plots of ${ }^{\mathrm{ANC}} c_{n}^{(J, j)}(\delta)$ for $j=1$ in the range $-10<{ }^{\mathrm{ANC}} c_{n}^{(J, j)}(\delta)<20$ which, (for a given $\delta$ ) includes classically open channels for $\varepsilon$ below 50 .

Three features should be mentioned:

(i) A nearly quadratic $\delta$-dependence for small $\delta$. This is the region of the fly-wheel (FW) approximation with dominating gyroscopic interaction.

(ii) A nearly linear $\delta$-dependence for large $\delta$. This is the region of the standard adiabatic channel (AC) approximation with dominating charge-dipole interaction..$^{2-4}$

(iii) Between these two regions, an interplay between the gyroscopic and electrostatic interactions is expected.

For very low energies, $\varepsilon \ll 1$, only channels with negative and small positive values of ${ }^{\mathrm{ANC}} c_{n}^{(J, j)}(\delta)$ contribute: this defines the ultra-low (UL) energy range. With increasing energies, a larger number of channels contribute: this defines

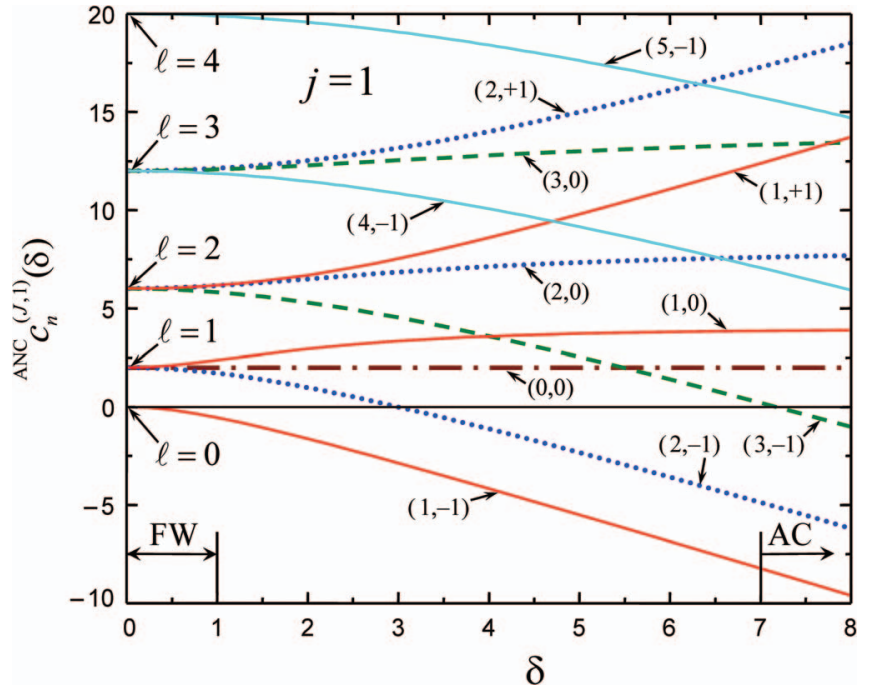

FIG. 2. Eigenvalues ${ }^{\mathrm{ANC}} c_{n}^{(J, j)}(\delta)$ of the interaction matrix for $j=1$ and $J=0$ (dashed-dotted brown line), $J=1$ (full red lines), $J=2$ (dotted blue lines), and $J=3$ (green dashed lines); $J=4$ (blue line) and $J=5$ (blue line), that contribute to the rate coefficients at low and medium energies up to $\varepsilon=50$. Curves are labeled by $(J, n)$ pairs. The ranges $\mathrm{FW}$ and AC indicate regions where the fly-wheel and adiabatic channel treatment are applicable.

an intermediate energy range. At high energies, the adiabatic channel approximation becomes valid.

In what follows, the results of calculation of the ANC rate coefficients are presented through the ratios in which $\mathrm{ACCl}$ rate coefficients are calculated analytically from Eqs. (3.1) and (3.2):

$$
\begin{aligned}
S^{(j)}(\varepsilon, \delta) & =\frac{{ }_{\mathrm{ANC}} \chi^{(j)}(\varepsilon, \delta)}{{ }_{\mathrm{ACCl}} \chi^{(j)}(\varepsilon, \delta)} \\
\text { and } \quad \bar{S}^{(j)}(\theta, \delta) & =\frac{{ }_{\mathrm{ANC}} \bar{\chi}^{(j)}(\theta, \delta)}{{ }_{\mathrm{ACCl}} \bar{\chi}^{(j)}(\theta, \delta)}
\end{aligned}
$$

As noted in Paper I, ${ }^{1}$ the qualitative behavior of $S^{(j)}(\varepsilon, \delta)$ and $\bar{S}^{(j)}(\theta, \delta)$, as functions of $\delta$, are characterized by a set of threshold values ${ }^{\mathrm{ANC}} \delta_{n}^{(J, j)}$ and a set of reference values ${ }^{\mathrm{R}} \delta_{n}^{(J, j)}$. Figure 3 shows the ratios $S^{(j)}\left(\varepsilon,{ }^{\mathrm{R}} \delta_{n}^{(J, j)}\right)$ for $j=1, j=2$, and 3 under conditions where either one or two capture channels are open at ultra-low energy (UL) energies (either threshold ${ }^{\mathrm{ANC}} \delta_{-j}^{(j, j)}$ or thresholds ${ }^{\mathrm{ANC}} \delta_{-j}^{(j, j)}$ and ${ }^{\mathrm{ANC}} \delta_{-j}^{(j+1, j)}$ ).

An interesting conclusion from Fig. 3 is that the rate coefficients ${ }^{\mathrm{ANC}} \chi^{(j)}\left(\varepsilon,{ }^{\mathrm{R}} \delta_{n}^{(J, j)}\right)$, at sufficiently large $\varepsilon$, oscillate about their mean value which is different from (though slowly approaching) their $\mathrm{ACCl}$ counterparts ${ }^{\mathrm{ACCl}} \chi^{(j)}\left(\varepsilon,{ }^{\mathrm{R}} \delta_{n}^{(J, j)}\right)$. The difference between the mean value of ${ }^{\mathrm{ANC}} \chi^{(j)}\left(\varepsilon,{ }^{\mathrm{R}} \delta_{n}^{(J, j)}\right)$ and ${ }^{\mathrm{ACCl}} \chi^{(j)}\left(\varepsilon,{ }^{\mathrm{R}} \delta_{n}^{(J, j)}\right)$ is due to the fact that the $\mathrm{ACCl}$ approximation completely ignores the gyroscopic interaction which, as seen from Fig. 2, has non-negligible effect even at high energies. Presumably, faster convergence of ${ }^{\mathrm{ANC}} \chi^{(j)}\left(\varepsilon,{ }^{\mathrm{R}} \delta_{n}^{(J, j)}\right)$ to its high-energy limit would be achieved if the latter was identified with ANCCl rate coefficient (see Paper $\mathrm{I}^{1}$ ). Had one used, as a reference rate coefficient, the AC quantum counterpart instead of the $\mathrm{ACCl}$ counterpart, the difference between ${ }^{\mathrm{ANC}} \chi^{(j)}\left(\varepsilon,{ }^{\mathrm{R}} \delta_{n}^{(J, j)}\right)$ and ${ }^{\mathrm{AC}} \chi^{(j)}\left(\varepsilon,{ }^{\mathrm{R}} \delta_{n}^{(J, j)}\right)$ would be larger. This is due to the fact that the $\mathrm{AC}$ approximation 


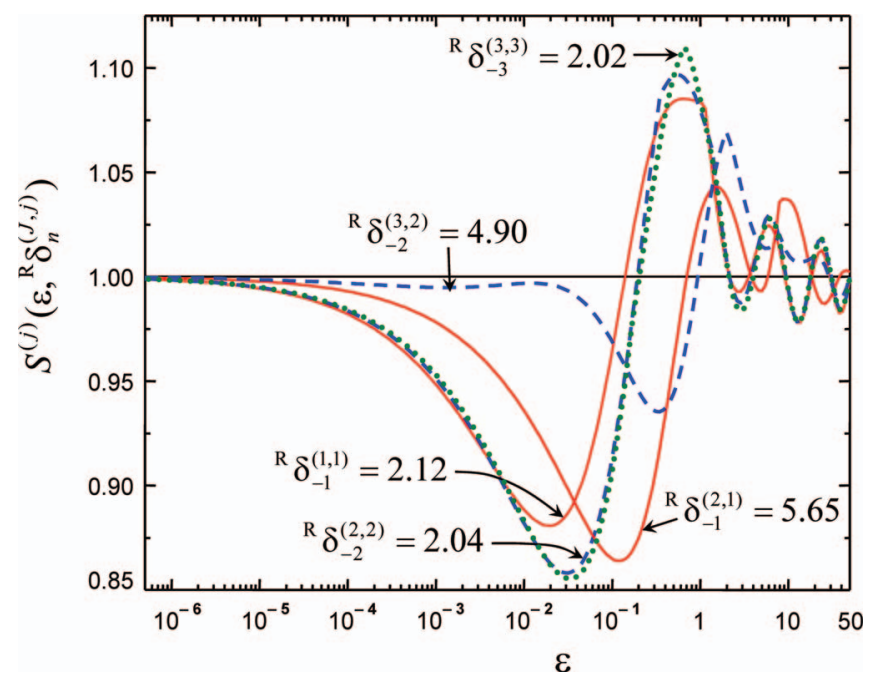

FIG. 3. Energy dependence of the ratios of axially nonadiabatic and classical adiabatic channel rate coefficients $S^{(j)}\left(\varepsilon,{ }^{\mathrm{R}} \delta_{n}^{(J, j)}\right)={ }^{\mathrm{ANC}} \chi^{(j)}\left(\varepsilon,{ }^{\mathrm{R}} \delta_{n}^{(J, j)}\right) /$ ${ }^{\mathrm{ACCl}} \chi^{(j)}\left(\varepsilon,{ }^{\mathrm{R}} \delta_{n}^{(J, j)}\right)$ for a set of reference values of reduced dipole moments $\mathrm{R}_{\delta_{n}}^{(J, j)}$ for $j=1,2,3$.

incorrectly attributes a non-zero centrifugal repulsion to the lower capture channel $J=j$, i.e., $\ell=0$. The ACCl approach artificially removes, by a series of approximations (see Paper $\mathrm{I}^{1}$ ), this inconsistency, thus producing a reasonable reference limit. Figure 4 illustrates the quenching of the undulations in $\bar{S}^{(1)}\left(\theta,{ }^{\mathrm{R}} \delta_{n}^{(J, j)}\right)$ as a result of the averaging of $S^{(j)}\left(\varepsilon,{ }^{\mathrm{R}} \delta_{n}^{(J, j)}\right)$.

Passing now to cases with $\delta$ different from ${ }^{\mathrm{R}} \delta_{n}^{(J, j)}$, we show in Fig. 5 the ratios $S^{(j)}(\varepsilon, \delta)$ for a set of $\delta$ values from the interval $0 \leq \delta \leq 4$.

The following properties of the low-energy range are noticed in Fig. 5:

(i) For $\delta=0,\left.S^{(1)}(\kappa, \delta)\right|_{\kappa \rightarrow 0} \rightarrow 2$ which is the Vogt-Wannier limit.

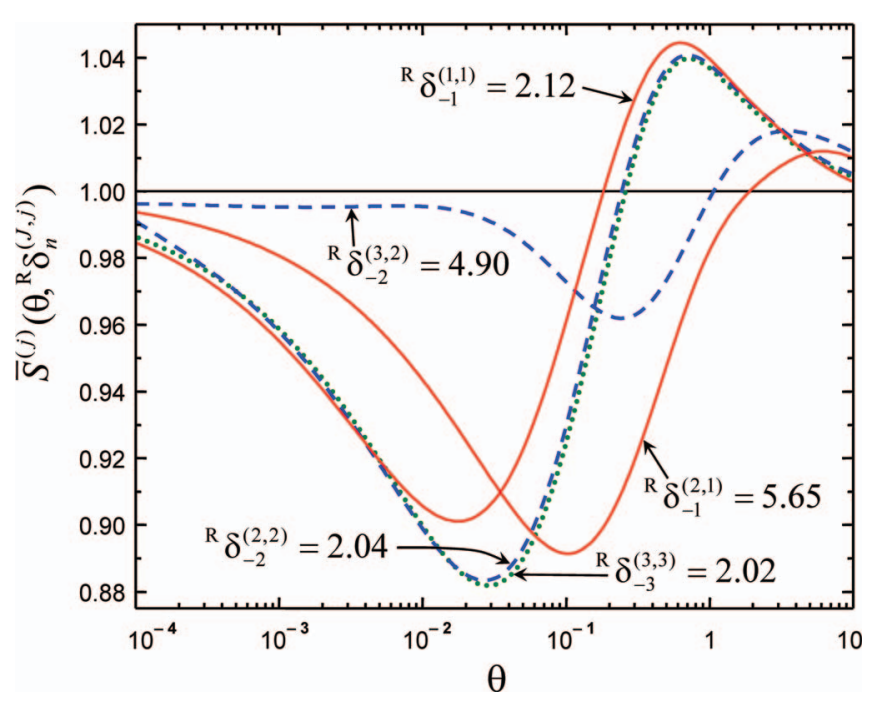

FIG. 4. Temperature dependence of the ratios of axially nonadiabatic and classical adiabatic channel rate coefficients $\bar{S}^{(j)}\left(\theta,{ }^{\mathrm{R}} \delta_{n}^{(J, j)}\right)={ }^{\mathrm{ANC}} \bar{\chi}^{(j)}$ $\left(\theta,{ }^{\mathrm{R}} \delta_{n}^{(J, j)}\right) /{ }^{\mathrm{ACCl}} \bar{\chi}^{(j)}\left(\theta,{ }^{\mathrm{R}} \delta_{n}^{(J, j)}\right)$ for a set of reference values ${ }^{\mathrm{R}} \delta_{n}^{(J, j)}$ with $j=1,2,3$, see Fig. 3 .

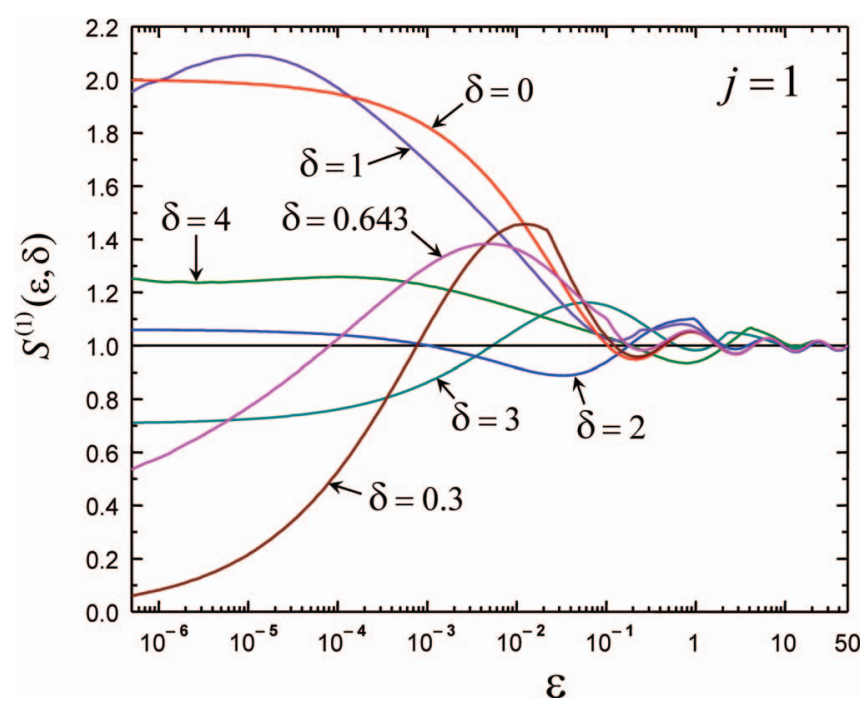

FIG. 5. Energy dependence of the ratios $S^{(1)}(\varepsilon, \delta)={ }^{\mathrm{ANC}} \chi^{(1)}(\varepsilon$, $\delta) /{ }^{\mathrm{ACCl}} \chi^{(1)}(\varepsilon, \delta)$ for $j=1$ and a regular set of $\delta$ with $0<\delta<4$.

(ii) For $\delta=0.3, S^{(1)}(\kappa, \delta)$ drops with decreasing $\kappa$. This compensates the too strong divergence of the $\mathrm{ACCl}$ rate in the limit $\kappa \rightarrow 0$ and brings the ANC rate to its FW counterpart.

(iii) For $\delta=1, S^{(1)}(\kappa, \delta)$ is about 2. This reflects the virtually complete opening of the first quantum channel in the ANC rate coefficient while its $\mathrm{ACCl}$ counterpart is smaller by a factor of 2 .

(iv) For $\delta=2, S^{(1)}(\kappa, \delta)$ is very close to unity. It would be exactly equal to unity for ${ }^{\mathrm{R}} \bar{\delta}_{-1}^{(1,1)}=2.12$.

(v) For $\delta=3$ and $\delta=4$, the $S^{(1)}(\kappa, \delta)$ lie on either side of the horizontal unity line. The values of $S^{(1)}(\kappa, \delta)$ replace the incorrect behavior of the $\mathrm{ACCl}$ rates in a situation where the ANC rates for $\delta=3$ and $\delta=4$ are almost the same.

In the intermediate energy range, the $S^{(j)}(\kappa, \delta)$ show the expected undulatory behavior that reflects the consecutive opening of capture channels with high values of the total angular momentum. Figure 6 illustrates the quenching of the undulations in $\bar{S}^{(1)}(\theta, \delta)$ as a result of the averaging of $S^{(1)}(\varepsilon, \delta)$.

The seemingly irregular dependence of $S^{(1)}(\varepsilon, \delta)$ and $\bar{S}^{(1)}(\theta, \delta)$ on $\delta$ at low energies and temperatures in Figs. 5 and 6 can be better understood by the study of the $\delta$ dependence of the ratios across a continuous range of $\delta$ at very low energy or temperature. Figure 7 presents plots of ${ }^{\mathrm{UL}} \bar{S}^{(j)}(\theta, \delta)$ for $j=1,2$, and 3 and $\theta=10^{-4}$ across a range of $\delta$ that accommodates several thresholds values, ${ }^{\mathrm{ANC}} \delta_{n}^{(J . j)}$, for opening of the CD capture channels and several reference values, ${ }^{\mathrm{R}} \delta_{n}^{(J . j)}$ where ${ }^{\mathrm{UL}} \bar{S}^{(j)}(\theta, \delta)$ crosses unity), see Table III.

Here, the vertical arrows mark the following thresholds: the first three arrows (collapsed into a single full black arrow) for the lowest channels with $J=j$, the second three (narrow spaced full, dashed, and dotted arrows) for the lowest channels with $J=j-1$, the third three (widely spaced dashed, dotted, and full arrows) for the lowest channels with $J=j+2$, the next two (dashed and dotted arrow) for the higher channels with $J=j$, and the last one (dashed arrow) 


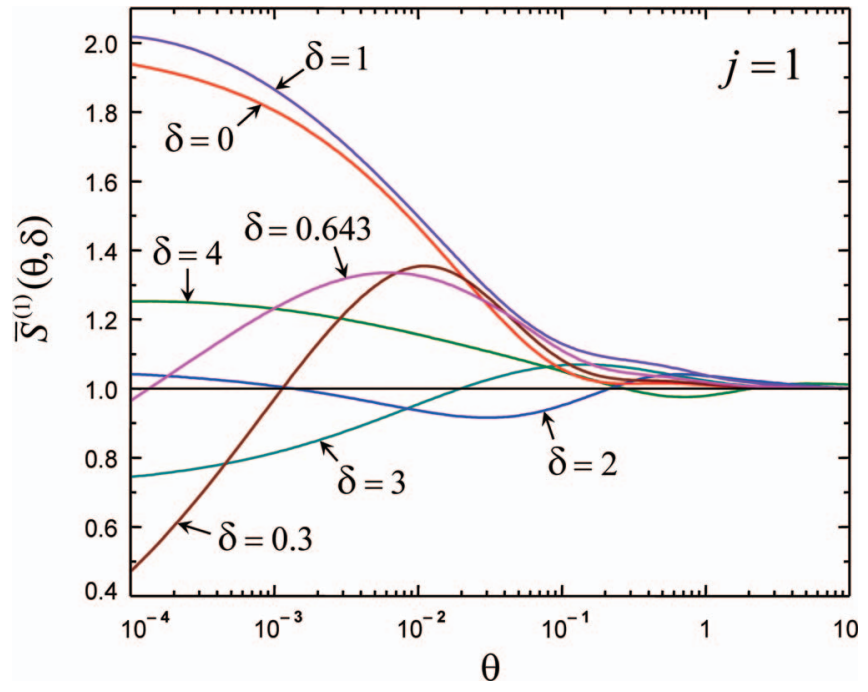

FIG. 6. Temperature dependence of the ratios $\bar{S}^{(1)}(\theta, \delta)={ }^{\mathrm{ANC}} \bar{\chi}^{(1)}(\theta, \delta) /$ ${ }^{\mathrm{ACCl}} \bar{\chi}^{(1)}(\theta, \delta)$ for $j=1$ and a set of different $\delta$ from the range $0<\delta$ $<4$.

for the second channel with $J=j=3$. The plots ${ }^{\mathrm{UL}} \bar{S}^{(j)}(\theta, \delta)$ explain low energy/temperature parts of Figs. 3-6, for those that exceed the first thresholds ${ }^{\mathrm{ANC}} \delta_{-j}^{(j, j)}$, where the ANC rates are not too different from their $\mathrm{ACCl}$ counterparts and where both rate coefficients display approximately the same temperature dependence, ${ }^{\mathrm{ANC}} \bar{\chi}^{(j)}(\theta, \delta) \propto{ }^{\mathrm{ACCl}} \bar{\chi}^{(j)}(\theta, \delta) \propto \theta^{-1 / 2}$. For $\delta<{ }^{\mathrm{ANC}} \delta_{-j}^{(J . j)}$, the ACCl and ANC rate coefficients show different temperature behavior in the UL range. They are, therefore, shown independently in Fig. 8 which is an extension of Fig. 1 of Ref. 10.

In this figure the UL region, for $\theta<5 \times 10^{-2}$ and $\delta$ $\leq 1$, corresponds to the FW approximation of Ref. 10 when only a single CD capture channel becomes completely open at $\delta \approx 1$. The FW s-wave rate coefficients (dotted blue lines) here are compared with their ANC (full red lines) and $\mathrm{ACCl}$

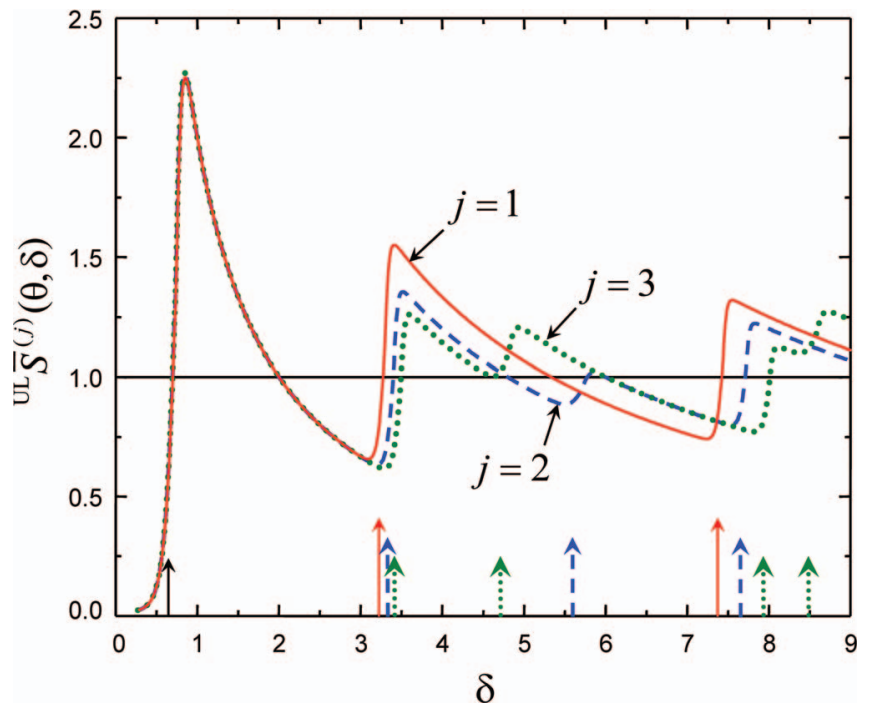

FIG. 7. Plots of ${ }^{\mathrm{UL}} \bar{S}^{(j)}(\theta, \delta)=\mathrm{ANCUL}_{\bar{\chi}}^{(j)}(\theta, \delta) / \mathrm{ANCUL}_{\bar{\chi}}^{(j)}(\theta, \delta)$ for $j=1$, 2 , and 3 and $\theta \approx 10^{-6}$. The arrows indicate thresholds for the opening of consecutive capture channels for pure $\mathrm{CD}$ interaction (see text for the assignment of the $\mathrm{CD}$ thresholds).

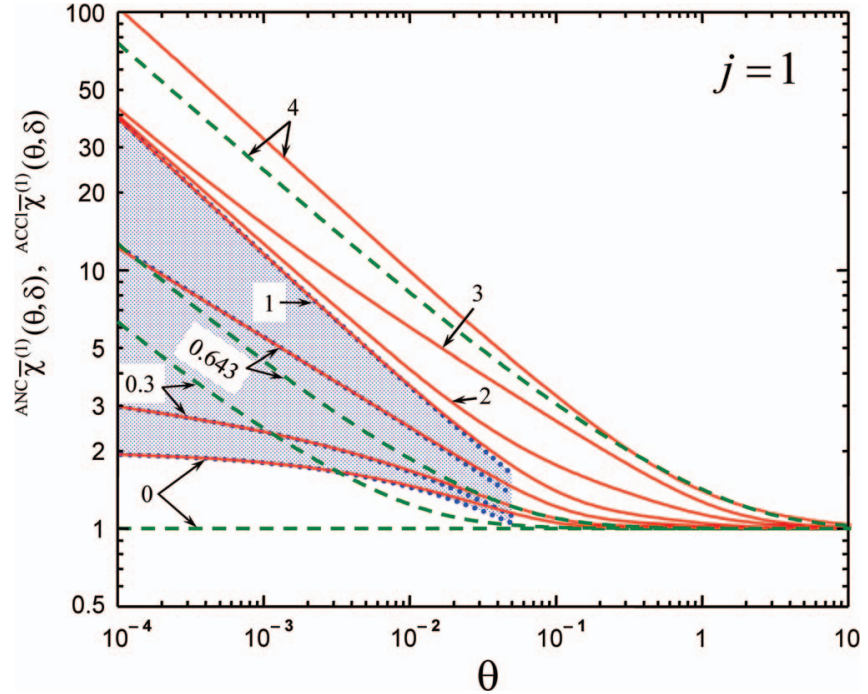

FIG. 8. Plots of ${ }^{\mathrm{ANC}} \bar{\chi}^{(1)}(\theta, \delta)$ (red full lines) and ${ }^{\mathrm{ACCl}} \bar{\chi}^{(1)}(\theta, \delta)$ (green dashed lines) from UL to higher temperatures and for a set of $\delta$. The marked region corresponds to the FW approximation of [10] (dotted blue lines).

(dashed green lines) counterparts. For $\delta<{ }^{\mathrm{ANC}} \delta_{-1}^{(1,1)}=0.643$, the ANC rate coefficients are close to the FW rate coefficients as given in Ref. 10 and they show a much slower increase with decreasing $\theta$ than the $\mathrm{ACCl}$ rate coefficients. The UL part of this figure helps to understand where a small dipole moment starts to modify the increase of the rate coefficient of a nonpolar molecule from the Vogt-Wannier limit. One sees for instance that, for $\delta=0.3$, the charge-dipole interaction noticeably modifies the charge-induced dipole rate coefficient at temperatures where the latter begins to switch from the Langevin to the Vogt-Wanner limit.

The UL temperature capture rate coefficients for $\delta<\delta_{-j}^{(j, j)},\left.{ }^{\text {ANC }} \bar{\chi}^{(j)}(\theta, \delta)\right|_{\theta \ll 1, \delta<{ }^{\mathrm{ANC}} \delta_{-j}^{(j, j)}}$ are obtained from the general expressions of Paper $\mathrm{I}^{1}$ which yield

$$
\begin{aligned}
& \left.{ }^{\mathrm{ANC}} \bar{\chi}^{(j)}(\theta, \delta)\right|_{\theta \ll 1, \delta<\mathrm{ANC}_{-j}^{(j, j)}} \\
& =(\theta / 8)^{\tau_{j}-1 / 2} \frac{\sin ^{2}\left(\pi \tau_{j}\right)}{\sqrt{\pi}} \frac{\Gamma^{2}\left(1-\tau_{j}\right)}{\Gamma\left(1+\tau_{j}\right)}\left[1+O\left((\theta / 8)^{\tau_{j}}\right)\right],
\end{aligned}
$$

where $\tau_{j}=\sqrt{1 / 4+\mathrm{ANC}_{c_{-j}}^{(j, j)}(\delta)}$. We note that the correction term $O(x)$ in Eq. (4.3) is small as long as $\tau_{j}$ is not too small, i.e., when $\delta$ is not too close to ${ }^{\mathrm{ANC}} \delta_{-j}^{(j, j)}$. As pointed in Paper I, ${ }^{1}$ the coefficients $\mathrm{ANC}_{c} c_{-j}^{(j, j)}(\delta)$, for $\delta<{ }^{\mathrm{ANC}} \delta_{-j}^{(j, j)}$, only weakly depend on $j$, and this dependence completely disappears in the limit $\delta \rightarrow 0$, as ${ }^{\mathrm{ANC}} c_{-j}^{(j, j)}(\delta)$ can be represented by $\left.{ }^{\mathrm{ANC}} c_{-j}^{(j, j)}(\delta)\right|_{\delta \ll 1}=-2 \delta^{2} / 3$. In this way, the zero-temperature limit of Eq. (4.3) for non-polar molecules passes to the VogtWannier capture rate coefficient:

$$
\begin{aligned}
\left.{ }^{\mathrm{ANC}} \bar{\chi}^{(j)}(\theta, \delta)\right|_{\theta \ll 1, \delta \ll 1} & =\left.{ }^{\mathrm{FW}} \bar{\chi}^{(j)}(\theta, \delta)\right|_{\theta \ll 1, \delta \ll 1} \\
& =2(\theta / 8)^{-2 \delta^{2} / 3}(1+O(\sqrt{\theta / 8}),
\end{aligned}
$$

$\left.{ }^{\mathrm{ANC}} \bar{\chi}^{(j)}(\theta, \delta)\right|_{\theta \rightarrow 0, \delta=0}=\left.{ }^{\mathrm{FW}} \bar{\chi}^{(j)}(\theta, \delta)\right|_{\theta \rightarrow 0, \delta=0} \rightarrow{ }^{\mathrm{VW}} \bar{\chi}=2$. 
For $\delta$ approaching $\delta_{-1}^{(1,1)}$, i.e., when the capture channel for pure $\mathrm{CD}$ interaction opens, the quantum rate coefficient for the full interaction (charge-dipole + charge-induced dipole) is close to the classical CD rate coefficient. We cannot judge whether this is accidental. Unfortunately, the method of calculating capture probabilities with the WKB approximation at small distances, does not allow one to trace the complete transition from the full interaction to the pure CD interaction. Indeed, with decreasing polarizability, the range of "small distances" shrinks, and one encounters similar difficulties as in the treatment of finite-size effects in electron capture. ${ }^{11}$ With a further increase of $\delta$, i.e., $\delta>\delta_{-j}^{(j, j)}$, several CD capture channels completely open up. The figure shows plots for one or two open channels for $\delta=1,2,3$, and 4 . The coincidence of the three rate coefficients with $\delta=1,2$, and 3 at $\theta=10^{-4}$ is a specific quantum effect when all contributions from higher waves, except for the leading one (for $j=1$, $J=1, n=-1)$ are totally suppressed. The rate coefficients in this case are $\left.{ }^{\mathrm{ANC}} \bar{\chi}^{(1)}(\theta, \delta)\right|_{\theta \rightarrow 0 ; \delta=1,2,3}=1 / \sqrt{2 \pi \theta}$. For $\delta=4$, the second channel with $j=1, J=2, n=-1$ opens, and $\left.{ }^{\mathrm{ANC}} \bar{\chi}^{(1)}(\theta, \delta)\right|_{\theta \rightarrow 0 ; \delta=4}=8 / 3 \sqrt{2 \pi \theta}$. On the other hand, the ANC and ACCl rate coefficients in the limit of small $\theta$ are given by ${ }^{\mathrm{ANCCD}} N^{(1)}(\delta) / \sqrt{2 \pi \theta}$ and ${ }^{\mathrm{ACClCD}} N^{(1)}(\delta) / \sqrt{2 \pi \theta}$, respectively, yielding the ratio $\left.\bar{S}^{(1)}(\theta, \delta)\right|_{\theta \rightarrow 0 ; \delta=4}$ $=\left.\left({ }^{\mathrm{ANC}} \bar{\chi}^{(1)}(\theta, \delta) /{ }^{\mathrm{ACCl}} \bar{\chi}^{(1)}(\theta, \delta)\right)\right|_{\theta \rightarrow 0 ; \delta=4}=\sqrt{2}$. With increasing $\theta$, the coincidence of ${ }^{A N C} \bar{\chi}^{(1)}(\theta, \delta)_{\delta=1,2,3}$ disappears and, e.g., the rate coefficients with $\delta=3$ approach those with $\delta=4$ at $\theta>10^{-1}$. For still larger $\theta$, the ANC rate coefficients converge to their $\mathrm{ACCl}$ counterparts as demonstrated by comparison of the upper red full line with the upper dotted green line, both corresponding to $\delta=4$.

\section{THE EFFECTS OF $K$-DOUBLING}

$K$-doubling manifests itself in a small splitting of the doubly degenerate state $j, k$ of a symmetric top rotor and arises as a result of comparably weak perturbations such as the coupling of rotation with doubly degenerate vibrations, spinorbital or hyperfine interactions. Accounting for $K$-doubling in capture dynamics complicates the analysis of the interplay between gyroscopic and electrostatic interactions, supplementing these by radial nonadiabatic coupling between the states of the collision complex that arise from the two $K$ doublet states of the free rotor. The main features of this interplay are discussed in the following.

The ANC states introduced in Paper $\mathrm{I}^{1}{ }^{\mathrm{ANC}}|J, j, n ; \delta\rangle$ are defined by those positive $\delta$ that correspond to a certain sign of the quantum number $k$ in the basis AC states $\left.\mathrm{AC}_{\mid J,} j, m, k\right\rangle$. The latter do not belong to a definite parity $p$ of the free rotor state and, therefore, the ANC states ${ }^{\mathrm{ANC}}|J, j, n ; \delta\rangle$ do not correspond to a definite total parity $P$ of the collision pair. If one uses the parity-adapted $\mathrm{AC}$ basis ${ }^{\mathrm{AC}}|J, j, m| k,|, p\rangle$ $=\frac{1}{\sqrt{2}}\left({ }^{\mathrm{AC}}|J, j, m, k\rangle+p^{\mathrm{AC}}|J, j, m,-k\rangle\right)$ for constructing the ANC matrix, its diagonalization yields the ANC states ${ }^{\mathrm{ANC}}|J, j, n, P ; \delta\rangle$ with certain total parity quantum number $P=(-1)^{\ell} p$, where $\ell$ is the quantum number of the relative angular momentum when it is well defined, i.e., in the limit $\delta \rightarrow 0$. The states ${ }^{\mathrm{ANC}}|J, j, n, P ; \delta\rangle$ and, therefore, the ANC potentials are doubly degenerate with respect to $P= \pm 1$.

The ANCK ( $\mathrm{K}$ for $K$-doubling) potentials are defined as eigenvalues of the interaction operator which is written as a generalization of Eqs. (2.1) and (2.2) of Paper I. ${ }^{1}$

$$
\hat{V}=\frac{(\hat{\mathbf{J}}-\hat{\mathbf{j}})^{2}}{2 \mu R^{2}}+\frac{q \mu_{D} \hat{k} \hat{\mathbf{j}} \mathbf{R}}{j(j+1) R^{3}}-\frac{q^{2} \alpha}{2 R^{4}}+\hat{p} \frac{\Delta E_{k}}{2} .
$$

Here the first term corresponds to the relative rotation, the second to the weak-field charge-dipole interaction, the third to the charge-induced dipole interaction, and the fourth is the $K$-doubling term with the splitting of $\Delta E_{k}$. The operators $\hat{k}$ and $\hat{p}$ do not commute: the former is diagonal in the $J, j, m, k$ representation with the eigenvalues $\pm|k|$, the latter in the $J, j$, $m,|k|, p$ representation with the eigenvalues \pm 1 . In this way, ANCK potentials are generated from ANC potentials by lifting the degeneracy with respect to $P$. In our reduced variables, a $J, j$ block of the operator $\hat{v}^{(J, j)}$ can be written as

$$
{ }^{\mathrm{ANCK}} \hat{v}^{(J, j)}(\rho, \delta)=\frac{{ }^{\mathrm{ANCK}} \hat{C}^{(J, j)}(\delta, \rho)}{2 \rho^{2}}-\frac{1}{2 \rho^{4}} .
$$

Here ${ }^{\mathrm{ANCK}} \hat{C}^{(J, j)}(\delta)$ is an operator

$$
{ }^{\mathrm{ANCK}} \hat{C}^{(J, j)}(\delta)=(\hat{\mathbf{J}}-\hat{\mathbf{j}})^{2}+2 \delta \hat{\mathbf{j}} \boldsymbol{\rho} / \rho \sqrt{j(j+1)}+\hat{p} \Delta \varepsilon \rho^{2} .
$$

The $\rho$-dependent eigenvalues of this operator, ${ }^{\mathrm{ANCK}} c_{n}^{(J, j, P)}(\delta, \rho)$, define the ANCK potentials

$$
{ }^{\mathrm{ANCK}} v_{n}^{(J, j, P)}(\delta, \rho)=\frac{{ }_{\mathrm{ANCK}} c_{n}^{(J, j, P)}(\delta, \rho)}{2 \rho^{2}}-\frac{1}{2 \rho^{4}} .
$$

Asymptotically, for $\delta \rightarrow 0$, the ${ }^{\mathrm{ANCK}} v_{n}^{(J, j, P)}(\delta, \rho)$ become

$$
\left.{ }^{\mathrm{ANCK}} v_{n}^{(J, j, P)}(\delta, \rho)\right|_{\delta \rightarrow 0} \rightarrow \frac{\ell(\ell+1)}{2 \rho^{2}}+p \frac{\Delta \varepsilon}{2}-\frac{1}{2 \rho^{4}} .
$$

The quantity $\ell \equiv \ell(J, j, n)$ of the r.h.s. of Eq. (5.5) is the relative angular momentum that corresponds to the triad $(J, j, n)$ of the l.h.s., while $p$ is the parity of the free top state. In this way we get

$$
P=(-1)^{\ell(J, j, n)} p .
$$

Depending on the characteristic value of the Massey parameter for the transition between $K$-doubling states, one can consider limiting cases of sudden and adiabatic motion across the ANCK potentials. If the Massey parameter is small, the nonadiabatic coupling corresponds to the sudden regime, and $K$-doubling effects can be ignored. This is the case for the $\mathrm{CH}_{3} \mathrm{D}$ molecule even for extremely low collision energies where $K$-doubling is due to hyperfine interaction and the splitting $\Delta \varepsilon$ falls into the $\mathrm{kHz}$ range. ${ }^{12}$ If the Massey parameter is large, nonadiabatic effects are small, and $K$-doubling effects show up. A large $K$-doubling effect can be expected, for instance, in the capture of $\mathrm{NO}\left(X^{2} \Pi_{1 / 2}\right)$ in different rotational states $j$ by an ion. Here the $\Lambda$-doubling splitting (more exactly, the doubling of the state with $\Omega=1 / 2$ ), which is due to second-order combined spin-orbit/rotational interaction with higher-lying electronic state, ${ }^{13}$ falls into the $\mathrm{MHz}$ range, ${ }^{14}$ and the two states $|j, \Omega=1 / 2, p\rangle$ with $p= \pm 1$ can be simulated (in terms of its wave-function) by two weakly split 
states $|j| k,|=1 / 2, p\rangle$ of a symmetric top with half-integer values of $j$. For the lowest rotational states $j=1 / 2, p= \pm 1$, the $4 \times 4$ matrix ${ }^{\mathrm{ANCK}} \mathbf{C}^{(J, 1 / 2)}$ can be diagonalized analytically to give the ANCK potentials

$$
\begin{aligned}
\operatorname{ANCK}_{v_{ \pm 1 / 2}^{(J, 1 / 2, P)}(\delta, \rho)=} & \frac{(J+1 / 2)^{2}}{2 \rho^{2}} \\
& \pm \sqrt{\left[\frac{\Delta \varepsilon}{2}-\sigma \frac{(J+1 / 2)}{2 \rho^{2}}\right]^{2}+\left(\frac{\delta}{\rho^{2} \sqrt{3}}\right)^{2}} \\
& -\frac{1}{2 \rho^{4}},
\end{aligned}
$$

where $\sigma$, the parity index, can assume values $\sigma= \pm 1$. Since $\ell(J, 1 / 2, \pm 1 / 2)=J \pm \sigma / 2$, from Eq. (5.6) we get

$$
P \equiv P_{ \pm 1 / 2, \sigma}^{(J, 1 / 2)}=(-1)^{\ell} p=(-1)^{J \pm \sigma / 2} p
$$

which relates the parity index $\sigma$ to the total parity quantum number $P$. The four states that correspond to four ANCK potentials in Eq. (5.7) form two pairs of states differing in $P$. The states within each pair are coupled by the radial nonadiabatic interaction. Special cases of Eq. (5.7) are the following:

(i) If $K$-doubling is neglected, i.e., for $\Delta \varepsilon=0$, Eq. (5.7) yields the corresponding ANC expression

$$
\begin{aligned}
\mathrm{ANC}_{v_{ \pm 1 / 2}^{(J, 1 / 2)}(\rho)=} & \frac{(J+1 / 2)^{2}}{2 \rho^{2}} \\
& \pm \sqrt{\left(\frac{(J+1 / 2)}{2 \rho^{2}}\right)^{2}+\left(\frac{\delta}{\rho^{2} \sqrt{3}}\right)^{2}} \\
& -\frac{1}{2 \rho^{4}} .
\end{aligned}
$$

In this case, four ANCK potentials from Eq. (5.7) collapse into two ANC potentials each being doubly degenerate with respect to the total parity $P= \pm 1$ and belonging to the same value of the intrinsic parity $p$. The nondegenerate states are mutually uncoupled, and the ANC expression in Eq. (5.9) can also be recovered from the ANCSC expression in Eq. (2.12) of Paper I, ${ }^{1}$ though the latter was derived under the assumption $J \gg 1$.

(ii) If Coriolis coupling is neglected, i.e., for $\sigma=0$, Eq. (5.7) yields the corresponding AN expression which accounts for $K$-doubling (ANK expression):

$$
\begin{aligned}
\operatorname{ANK}_{ \pm 1 / 2}^{(J, 1 / 2)}(\rho)= & \frac{(J+1 / 2)^{2}}{2 \rho^{2}} \\
& \pm \sqrt{\left(\frac{\Delta \varepsilon}{2}\right)+\left(\frac{\delta}{\rho^{2} \sqrt{3}}\right)^{2}}-\frac{1}{2 \rho^{4}}
\end{aligned}
$$

In this case, four ANCK potentials from Eq. (5.7) collapse into two ANC potentials each being doubly degenerate with respect to the total parity $P= \pm 1$ and belonging to different values of the intrinsic parity $p$. The non-degenerate states belonging to the same $P$ are coupled by the radial nonadiabatic interaction. This coupling was discussed in Ref. 15 in a description of its lambda-doublet
TABLE I. Dipole moments of $\mathrm{CH}_{3} \mathrm{D}$ and $\mathrm{CHD}_{3}$.

\begin{tabular}{lcccc}
\hline \hline Reference & $\begin{array}{c}\mathrm{CH}_{3} \mathrm{D}, \\
10^{-3} \mathrm{D}\end{array}$ & $\begin{array}{c}\mathrm{CH}_{3} \mathrm{D}, \\
10^{-3} \text { a.u. }\end{array}$ & $\begin{array}{c}\mathrm{CHD}_{3}, \\
10^{-3} \mathrm{D}\end{array}$ & $\begin{array}{c}\mathrm{CHD}_{3}, \\
10^{-3} \text { a.u. }\end{array}$ \\
\hline Expt. $\mu_{D}^{(a)} 16$ & 4.1 & 1.61 & 4.3 & 1.69 \\
Expt. $\mu_{D}^{(b)} 17$ & $5.57 \pm 0.10$ & $2.19 \pm 0.04$ & $5.69 \pm 0.14$ & $2.24 \pm 0.06$ \\
Theor. $\mu_{D}^{(c)} 17$ & $6.8 \pm 0.5$ & $2.7 \pm 0.2$ & $6.6 \pm 0.4$ & $2.6 \pm 0.2$ \\
\hline \hline
\end{tabular}

specificity in the low-temperature capture of $\operatorname{NO}\left(X^{2} \Pi_{1 / 2}\right)$ in its lowest rotational state by ions.

In the general case, the ANCK potentials from Eq. (5.7) provide a general access to an analytical treatment of the parity-changing nonadiabatic transitions between two $\Lambda$-doubling states of diatomic molecules in degenerate electronic states for $j=1 / 2$.

\section{A CASE STUDY: CAPTURE OF $\mathrm{CH}_{3}$ D AND $\mathrm{CD}_{3} \mathrm{H}$ IN DIFFERENT ROTATIONAL STATES BY $\mathrm{H}^{+}, \mathrm{D}^{+}$, AND $\mathrm{H}_{3}{ }^{+}$}

As indicated in Sec. $\mathrm{V}, \mathrm{K}$-doubling in $\mathrm{CH}_{3} \mathrm{D}$ is very small, and its effect on capture can be neglected down to very low temperatures, i.e., below $10^{-6} \mathrm{~K}$. We, therefore, use the results of Sec. IV for a calculation of capture rate coefficients ignoring $K$-doubling effects. In this case, the capture of $\mathrm{CH}_{3} \mathrm{D}$ and $\mathrm{CD}_{3} \mathrm{H}$ in different rotational states $j, k$ by $\mathrm{H}^{+}$, $\mathrm{D}^{+}$, and $\mathrm{H}_{3}{ }^{+}$provides an instructive illustration of the interplay of electrostatic and gyroscopic interactions in the capture dynamics. This is facilitated by the fact that the values of $\delta=\delta(j, k)$ for the dipole moments $\mu_{D}$ of $\mathrm{CH}_{3} \mathrm{D}$ and $\mathrm{CD}_{3} \mathrm{H}$ (see Table I) are close to the value of $\bar{\delta}_{c}^{(j)}$ discussed in Sec. IV. In what follows, we concentrate on collisions between $\mathrm{CH}_{3} \mathrm{D}(j, k)$ and $\mathrm{H}^{+}$and later consider other ions.

Values of $\delta(j, k)$ with $j=1, k=1$, and $j=2, k=1$ and 2 , for $\mathrm{CH}_{3} \mathrm{D}(j, k)+\mathrm{H}^{+}$capture, and the relevant dipole moments are listed in Table II.

Figure 9 shows plots of ANC rate coefficients for $j=1$, ${ }^{\text {ANC }} \bar{\chi}^{(1)}(\theta, \delta)$ and three values of the dipole moments. Also shown are the Langevin rate coefficient (dashed line), and its quantum counterpart $\left.{ }^{\mathrm{ANC}} \bar{\chi}^{(1)}(\theta, \delta)\right|_{\delta=0}$ (dotted line) tending, in the limit of low temperatures, to the Vogt-Wannier limit.

TABLE II. Values of reduced dipole moments $\delta^{(x)}(j, k)$ for capture of $\mathrm{H}^{+}$ by $\mathrm{CH}_{3} \mathrm{D}(j, k)$ in various rotational states $j, k$ (for the three dipole moments $\mu_{D}^{(a)}, \mu_{D}^{(b)}, \mu_{D}^{(c)}$ of Table I).

\begin{tabular}{llll}
\hline \hline$j, k$ & $\delta^{(a)}$ & $\delta^{(b)}$ & $\delta^{(c)}$ \\
\hline 1,1 & 1.98 & 2.69 & 3.28 \\
2,1 & 1.14 & 1.55 & 1.89 \\
2,2 & 2.28 & 3.10 & 3.78 \\
3,1 & 0.807 & 1.10 & 1.33 \\
3,2 & 1.61 & 2.20 & 2.68 \\
3,3 & None & None & None \\
4,1 & 0.625 & 0.849 & 1.04 \\
4,2 & 1.25 & 1.70 & 2.07 \\
4,3 & 1.88 & 2.55 & 3.11 \\
4,4 & 2.50 & 3.40 & 4.15 \\
\hline \hline
\end{tabular}




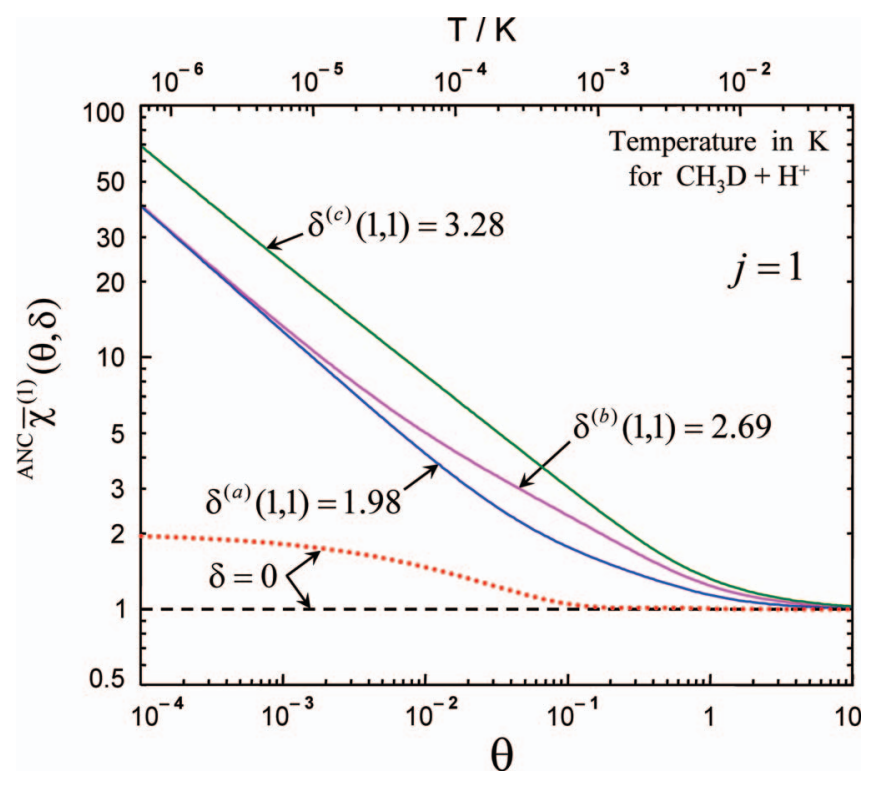

FIG. 9. Plots of ${ }^{\mathrm{ANC}} \bar{\chi}^{(1)}(\theta, \delta)$ for capture $\mathrm{CH}_{3} \mathrm{D}(j=1, k=1)+\mathrm{H}^{+}$(full curves, labeled by three values of $\delta=\delta^{(x)}(j, k)$, that correspond to the three possible values of $\mu_{D}$ of Table I). The plot of $\left.{ }^{A N C} \bar{\chi}^{(1)}(\theta, \delta)\right|_{\delta=0}$ (dotted red line) and the Langevin limit (dashed black line) are also shown.

Together with the scaled temperature $\theta$, here also the conventional temperature $T(\mathrm{~K})$ is given. The low-temperature convergence of the plots with $\delta^{(a)}=1.98$ and $\delta^{(b)}=2.69$ parallels that for $\delta=2$ and $\delta=3$ in Fig. 6, while the plot with $\delta^{(c)}=3.28$ lies noticeably higher than that for $\delta^{(b)}=2.69$. This is due to the fact that the value of $\delta^{(c)}=3.28$ is above the threshold value ${ }^{\mathrm{ANC}} \delta_{-1}^{(2,1)}=3.23$ that corresponds to the full opening of the second capture channel for pure CD interaction. For the former case, with charge-dipole and chargeinduced dipole interaction, the second channel is only partially open. This observation is consistent with Fig. 7 which

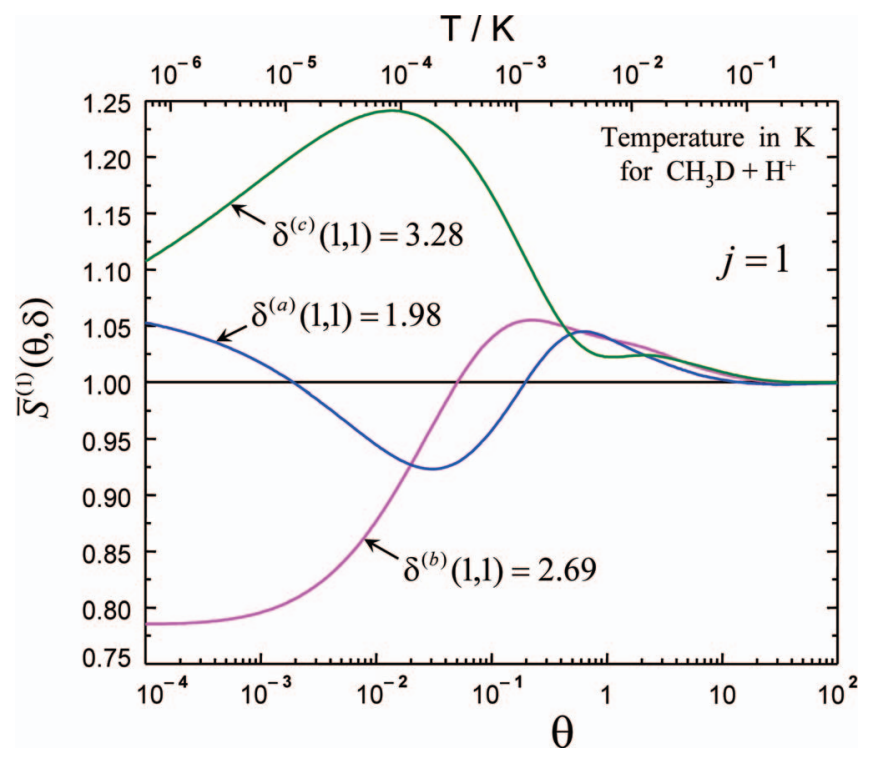

FIG. 10. Ratios $\bar{S}^{(1)}(\theta, \delta)={ }^{\mathrm{ANC}} \bar{\chi}^{(1)}(\theta, \delta) /{ }^{\mathrm{ACCl}} \bar{\chi}^{(1)}(\theta, \delta)$ for capture $\mathrm{CH}_{3} \mathrm{D}(j=1, k=1)+\mathrm{H}^{+}$(full curves, labeled by three values of $\delta=\delta^{(x)}(1$, 1 ), that correspond to the three possible values of $\mu_{D}$ of Table I).

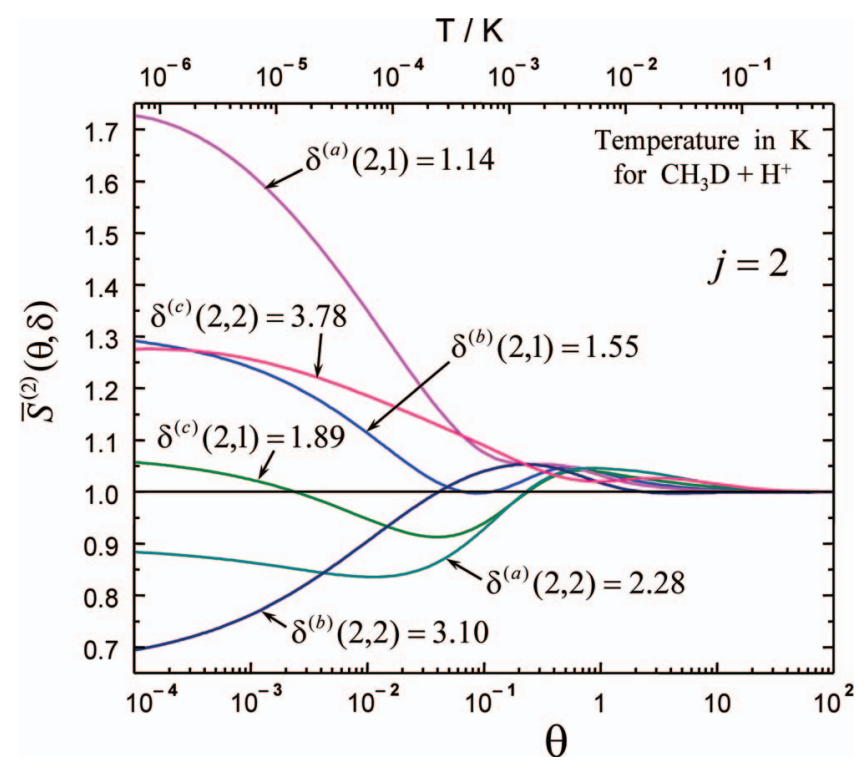

FIG. 11. Ratios $\bar{S}^{(2)}(\theta, \delta)={ }^{\mathrm{ANC}} \bar{\chi}^{(2)}(\theta, \delta) /{ }^{\mathrm{ACCl}} \bar{\chi}^{(2)}(\theta, \delta)$ for $\mathrm{CH}_{3} \mathrm{D}(j=2$, $k=1,2)+\mathrm{H}^{+}$capture (full curves labeled by $\delta=\delta^{(x)}(j, k)$, that correspond to the three possible values of $\mu_{D}$ of Table I).

shows a steep increase of the UL capture probability across the range $3.23<\delta<3.5$.

A comparison of ANC and $\mathrm{ACCl}$ rate coefficients is illustrated in Figs. 10 and 11 by plots of the ratios $\bar{S}^{(1)}\left(\theta, \delta^{(x)}\right)$ and $\bar{S}^{(2)}\left(\theta, \delta^{(x)}\right)$.

Here the positions of the plots in their UL energy part are consistent with the order of the parameters $\delta$ (see Table III) given by

$$
\begin{gathered}
{ }^{\mathrm{ANC}} \delta_{-1}^{(1,1)}<\delta^{(a)}(1,1)<{ }^{\mathrm{R}} \delta_{-1}^{(1,1)}<\delta^{(b)}(1,1) \\
<{ }^{\mathrm{ANC}} \delta_{-1}^{(2,1)}<\delta^{(c)}(1,1), \\
{ }^{\mathrm{ANC}} \delta_{-2}^{(2,2)}<\delta^{(a)}(2,1)<\delta^{(b)}(2,1)<\delta^{(c)}(2,1)<{ }^{\mathrm{R}} \delta_{-2}^{(2,2)} \\
<\delta^{(a)}(2,2)<\delta^{(b)}(2,2)<{ }^{\mathrm{ANC}} \delta_{-2}^{(3,2)}<\delta^{(c)}(2,2) .
\end{gathered}
$$

One notices that the $\mathrm{ACCl}$ approximation, extrapolated from higher energies into the UL range, performs rather satisfactorily, with maximum deviations of about $20 \%$ in the UL range. Larger differences between ${ }^{\mathrm{ANC}} \bar{\chi}^{(j)}(\theta, \delta)$ and ${ }^{\mathrm{ACCl}} \bar{\chi}^{(j)}(\theta, \delta)$ begin to show up in the range $0.1<\theta<1$. For the system under discussion, with the parameters $\alpha\left(\mathrm{CH}_{3} \mathrm{D}\right)$ $=17.3$ a.u. and $\mu\left(\mathrm{CH}_{3} \mathrm{D}+\mathrm{H}^{+}\right)=1.7 \times 10^{3}$ a.u., this range

TABLE III. Threshold values ${ }^{\mathrm{ANC}} \delta_{n}^{(J, j)}$ (for the abrupt opening of the capture channel $(J, j, n)$ for pure CD interaction) and reference values ${ }^{\mathrm{R}} \delta_{n}^{(J, j)}$.

\begin{tabular}{cccccc}
\hline \hline & $\mathrm{ANC}_{\delta_{-j}^{(j, j)}}$ & $\mathrm{ANC}_{\delta_{-j}^{(j+1, j)}}$ & $\mathrm{ANC}_{\delta_{-j+1}^{(j-1, j)}}$ & $\mathrm{ANC}_{-j}^{(j+2, j)}$ & $\mathrm{ANC}_{\delta_{-j+1}^{(j, j)}}$ \\
$\mathrm{j}$ & $\mathrm{R}_{\delta_{-j}^{(j, j)}}$ & $\mathrm{R}_{-j}^{(j+1, j)}$ & $\mathrm{R}_{\delta_{-j+1}^{(j-1, j)}}$ & $\mathrm{R}_{-j}^{(j+2, j)}$ & $\mathrm{R}_{-j+1}^{(j, j)}$ \\
\hline 1 & 0.643 & 3.23 & $\ldots$ & 7.38 & $\ldots$ \\
& 2.12 & 5.65 & $\ldots$ & 10.6 & $\ldots$ \\
2 & 0.641 & 3.34 & 5.60 & 7.66 & 9.88 \\
& 2.04 & 4.90 & 6.12 & 9.80 & 11.84 \\
3 & 0.640 & 3.42 & 4.72 & 7.94 & 8.49 \\
& 2.02 & 4.62 & 6.06 & $\ldots$ & 11.26 \\
\hline \hline
\end{tabular}


TABLE IV. Reduced dipole moments $\delta$ and scaling temperatures $T_{L}=\left(E_{L} / k_{\mathrm{B}}\right)$ for capture of isotopically substituted methane molecules by various ions, calculated with dipole moments $\mu_{D}^{(a)}, \mu_{D}^{(b)}$, and $\mu_{D}^{(c)}$ for $\mathrm{CH}_{3} \mathrm{D}$ and $\mathrm{CD}_{3} \mathrm{H}$ from Table I (with $\alpha\left(\mathrm{CH}_{3} \mathrm{D}\right)=\alpha\left(\mathrm{CD}_{3} \mathrm{H}\right)=\alpha\left(\mathrm{CH}_{4}\right)=17.3$ a.u. $=2.56 \mathrm{~A}^{18}$ ).

\begin{tabular}{lcc}
\hline \hline Collision & $\delta$ & $T_{\mathrm{L}} / 10^{-3} \mathrm{~K}$ \\
\hline $\mathrm{CH}_{3} \mathrm{D}+\mathrm{H}^{+}$ & $1.98 ; 2.69 ; 3.28$ & 6.08 \\
$\mathrm{CH}_{3} \mathrm{D}+\mathrm{D}^{+}$ & $3.75 ; 5.08 ; 6.21$ & 1.69 \\
$\mathrm{CH}_{3} \mathrm{D}+\mathrm{H}_{3}^{+}$ & $5.33 ; 7.25 ; 8.85$ & 0.834 \\
$\mathrm{CHD}_{3}+\mathrm{H}^{+}$ & $2.09 ; 2.76 ; 3.20$ & 6.00 \\
$\mathrm{CHD}_{3}+\mathrm{D}^{+}$ & $3.97 ; 5.25 ; 6.10$ & 1.65 \\
$\mathrm{CHD}_{3}+\mathrm{H}_{3}^{+}$ & $5.68 ; 7.52 ; 8.73$ & 0.808 \\
\hline \hline
\end{tabular}

of $\theta$ according to Eq. (2.1) with $T_{L}=\left(E_{L} / k_{\mathrm{B}}\right)=6.08 \times 10^{-3} \mathrm{~K}$ corresponds to $T$ between $10^{-3}$ and $10^{-2} \mathrm{~K}$. Values of $\delta$ and $T_{L}$ for other collision partners are listed in Table IV.

A summary of ANC capture rate coefficients for $j=1$, $k=1$ states of $\mathrm{CH}_{3} \mathrm{D}$ and $\mathrm{CD}_{3} \mathrm{H}$ in collisions with different ionic partners is presented in Fig. 12 where $\bar{S}^{(1)}\left(\theta, \delta^{(x)}\right)$ is shown for $\theta=10^{-4}$.

It should be finally noted that the importance of the gyroscopic effect for the capture of $\mathrm{CH}_{3} \mathrm{D}$ molecules shows the large difference between the threshold values of the ANC and AC reduced dipole moments, ${ }^{\mathrm{ANC}} \delta_{-j}^{(j, j)}$ and ${ }^{\mathrm{AC}} \delta_{-j}^{(j, j)}$ $=(j+1 / 8) \sqrt{j(j+1)} / j$. For instance, two values of $\delta^{(x)}(j, k)$ for $j=2, \delta^{(a)}(2,1)=1.14$ and $\delta^{(b)}(2,1)$ $=1.55$ are above the first ANC threshold ${ }^{\mathrm{ANC}} \delta_{-2}^{(2,2)}=0.641$ but are below the first $\mathrm{AC}$ threshold ${ }^{\mathrm{AC}} \delta_{-2}^{(2,2)}=2.60$, and all values of $\delta^{(x)}(j, k)$ for $j=3$ are above the first ANC threshold ${ }^{\mathrm{ANC}} \delta_{-3}^{(3,3)}=0.640$ but are below the first AC threshold ${ }^{{ }^{A C}} \delta_{-3}^{(3,3)}=3.61$ (see Table I from Paper $\left.\mathrm{I}^{1}\right)$. This implies that the AC approximation, which ignores the Coriolis coupling, is completely inadequate for an estimation of the lowtemperature capture rate coefficients for the considered states

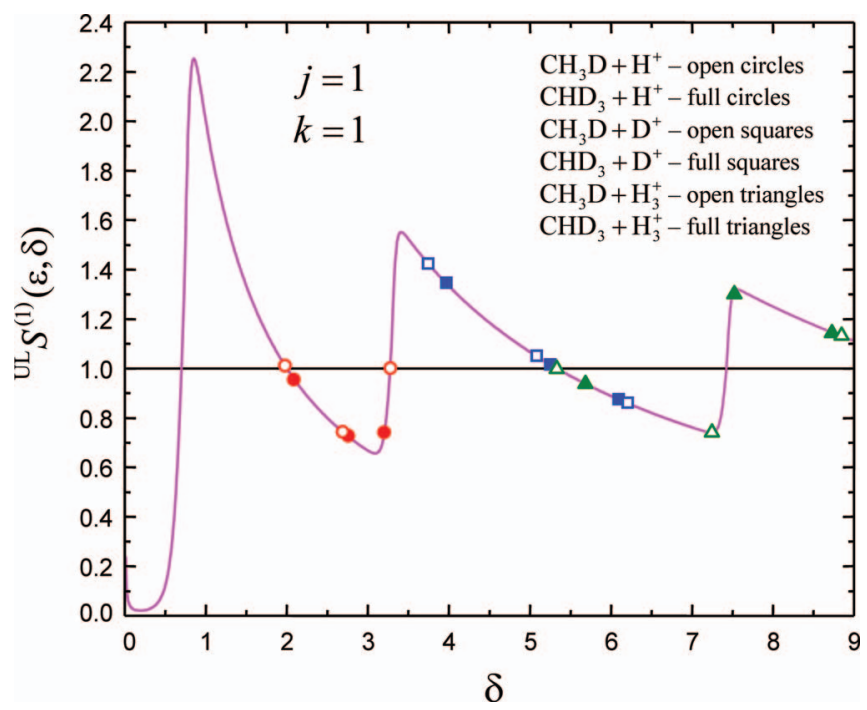

FIG. 12. Ratios ${ }^{\mathrm{UL}} S^{(1)}(\varepsilon, \delta)$ for the capture $\mathrm{CH}_{3} \mathrm{D}+\mathrm{H}^{+}$(open circles), $\mathrm{CD}_{3} \mathrm{H}+\mathrm{H}^{+}$(filled circles), $\mathrm{CH}_{3} \mathrm{D}+\mathrm{D}^{+}$(open squares), $\mathrm{CD}_{3} \mathrm{H}+\mathrm{D}^{+}$(filled squares), $\mathrm{CH}_{3} \mathrm{D}+\mathrm{H}_{3}{ }^{+}$(open triangles), $\mathrm{CD}_{3} \mathrm{H}+\mathrm{H}^{+}$(filled triangles) $+\mathrm{H}^{+}$ superimposed onto the plots ${ }^{\mathrm{UL}} S^{(j)}(\varepsilon, \delta)$ for $\theta=10^{-6}$ (full line). of the $\mathrm{CH}_{3} \mathrm{D}$ molecules. Here, the $\mathrm{ACCl}$ approach performs much better, since, as a consequence of the classical approximations, to a large extent it mimics the gyroscopic interaction.

\section{CONCLUSION}

This article has served two purposes. First, it provided the quantitative link between the two limiting ranges of capture between the gyroscopic- dominated and the electrostaticdominated collision regimes. For values of $\delta<1$, the capture dynamics is described by the fly-wheel approximation if the collision energy or temperature is small $(\varepsilon \ll 1, \theta \ll 1)$ while, for $\delta>1$, the standard adiabatic channel approximation can be used if the collision energy or temperature is large $(\varepsilon \gg 1$, $\theta \gg 1)$. In the wide range of intermediate cases the general ANC approach formulated in Paper $\mathrm{I}^{1}$ applies.

Second, it illustrated the magnitude of the treated effects by considering the capture of an ion by a symmetric top with a small dipole moment which can be obtained by isotopic substitution of an atom in a spherical top molecule. This was illustrated by the capture of ions by substituted methane molecules. For $\mathrm{CH}_{3} \mathrm{D}+\mathrm{H}^{+}, \delta$ varies (for different rotational states and different possible values of the dipole moment of $\mathrm{CH}_{3} \mathrm{D}$ ) between values above and close to unity, implying a competing manifestation of gyroscopic and electrostatic interactions in the capture dynamics. Here, the ultra-low temperature range, where not too many capture channels contribute to the rate coefficient, corresponds to temperatures below $10^{-2} \mathrm{~K}$. This is an important reference situation, since $\delta$ might be larger if the proton is replaced by heavier ions, or lower if the $\mathrm{CH} / \mathrm{CD}$ moiety is replaced by an $\mathrm{AB} / \mathrm{AB}^{\prime}$ moiety with smaller change in the reduced mass upon substitution.

Unfortunately, at present there are no experimental data for capture under the conditions discussed here. Nonetheless, we hope that our work motivates low-temperature capture studies under conditions where very small dipole moments of the neutral can substantially change the capture rate coefficients in comparison to those for nonpolar molecules.

\section{ACKNOWLEDGMENTS}

Financial support of this work by the EOARD Grant Award FA 8655-11-1-3077 is gratefully acknowledged.

\footnotetext{
${ }^{1}$ M. Auzinsh, E. I. Dashevskaya, I. Litvin, E. E. Nikitin, and J. Troe, J. Chem. Phys. 139, 084311 (2013).

2J. Troe, J. Chem. Phys. 87, 2773 (1987).

${ }^{3}$ S. C. Smith and J. Troe, J. Chem. Phys. 97, 5451 (1992).

${ }^{4}$ J. Troe, J. Chem. Phys. 105, 6249 (1996).

${ }^{5}$ E. I. Dashevskaya, I. Litvin, E. E. Nikitin, I. Oref, and J. Troe, J. Phys. Chem. A, 108, 8703 (2004).

${ }^{6}$ E. E. Nikitin and J. Troe, J. Phys. Chem. A 114, 9762 (2010).

${ }^{7}$ M. Auzinsh, E. I. Dashevskaya, I. Litvin, E. E. Nikitin, and J. Troe, J. Phys. Chem. A 115, 5027 (2011).

${ }^{8}$ E. I. Dashevskaya, I. Litvin, E. E. Nikitin, and J. Troe, Mol. Phys. 108, 873 (2010).

${ }^{9}$ L. D. Landau and E. M. Lifshitz, Quantum Mechanics (Pergamon Press, Oxford, 1977).

${ }^{10}$ M. Auzinsh, E. I. Dashevskaya, E. E. Nikitin, and J. Troe, Mol. Phys. 111, 2003 (2013).

${ }^{11}$ E. E. Nikitin and J. Troe, Phys. Chem. Chem. Phys. 12, 9011 (2010).
} 
${ }^{12}$ S. C. Wofsy, J. S. Muenter, and W. Klemperer, J. Chem. Phys. 53, 4005 (1970).

${ }^{13}$ R. N. Zare, Angular Momentum (Wiley, New York, 1988).

${ }^{14}$ W. L. Meerts and A. Dymanus, J. Mol. Spectrosc. 44, 320 (1972).

${ }^{15}$ M. Auzinsh, E. I. Dashevskaya, I. Litvin, E. E. Nikitin, and J. Troe, J. Chem. Phys. 130, 014304 (2009).
${ }^{16}$ R. Böhmer, U. Giebenhain, and A. Loidl, Mol. Phys. 82, 531 (1994).

${ }^{17}$ H. Hollenstein, R. R. Marquardt, M. Quack, and M. A. Suhm, J. Chem. Phys. 101, 3588 (1994).

${ }^{18}$ A. A. Radzig and B. M. Smirnov, Reference Data on Atoms, Molecules, and Ions, Springer Series in Chemical Physics Vol. 31 (Springer, Berlin, 1985). 